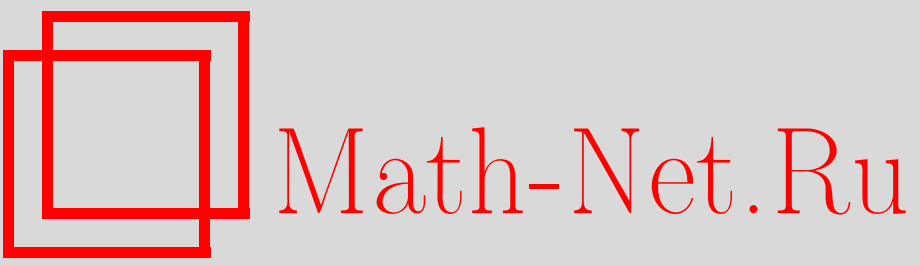

Г. А. Айгунов, Об ограниченности ортонормированных собственных функций одного класса операторов Штурма-Лиувилля с весовой функцией неограниченной вариациина конечном отрезке, УМH, 1996, том 51, выпуск 2, 143-144

DOI: https://doi.org/10.4213/rm947

Использование Общероссийского математического портала Math-Net.Ru подразумевает, что вы прочитали и согласны с пользовательским соглашением

http://www.mathnet.ru/rus/agreement

Параметры загрузки:

IP : 3.93 .64 .190

26 апреля 2023 г., $16: 32: 56$ 


\title{
ОБ ОГРАНИЧЕННОСТИ ОРТОНОРМИРОВАННЫХ СОБСТВЕННЫХ ФУНКЦИЙ ОДНОГО КЛАССА ОПЕРАТОРОВ ШТУРМА-ЛИУВИЛЛЯ С ВЕСОВОЙ ФУНКЦИЕЙ НЕОГРАНИЧЕННОЙ ВАРИАЦИИ НА КОНЕЧНОМ ОТРЕЗКЕ
}

\author{
Г. А. АЙгунов
}

1. Пусть $0<m \leqslant M$ - фиксированные числа. Обозначим через $Q$ множество всех измеримых на сегменте $[0,1]$ функций $\rho(x)$, удовлетворяющих условию

$$
0<m \leqslant \rho(x) \leqslant M<\infty .
$$

Пусть $\rho(x) \in Q$, рассмотрим спектральную задачу

$$
\begin{aligned}
& -y^{\prime \prime}(x)=\lambda \rho(x) y(x) \quad(0<x<1) \\
& y(0)=y(1)=0 \\
& \int_{0}^{1} \rho(x) y^{2}(x) d x=1 .
\end{aligned}
$$

Известно, что спектр задачи (1)-(3) дискретен и состоит из простых положительных собственных чисел $\lambda_{n}(n=1,2, \ldots)$. Обозначим через $u_{n}(x)$ собственную функцию, соответствующую собственному числу $\lambda_{n}$. Условимся говорить, что набор собственных функций $u_{n}(x)$ $(n=1,2, \ldots)$ ассоциирован с весовой функцией $\rho(x)$ спектральной задачи $(1)-(3)$.

В классических работах Штурма, Лиувилля и Стеклова в предположении некоторой гладкости весовой функции $\rho(x)$ было доказано, что

$$
\sup _{n} \max _{0 \leqslant x \leqslant 1}\left|u_{n}(x)\right| \leqslant C_{0}<\infty, \quad C_{0}>0 .
$$

В 1983 г. было выяснено [1], что в классе всех весовых функций $\rho(x) \in Q$ неравенство (4), вообще говоря, неверно.

Во многих вопросах анализа представляет значительный интерес выяснение условий, при которых собственные функции задачи (1)-(3) ограничены в совокупности, т.е. выполнено условие (4). В работах [2]-[3] были указаны достаточные условия, обеспечивающие выполнение неравенства (4). В частности, в [3] было выяснено, что если весовая функция $\rho(x)$ является функцией ограниченной вариации, то справедливо неравенство (4).

Цель настоящей работы - указать класс весовых функций неограниченной вариации $Q_{1}$ таких, что для собственных функций спектральной задачи (1)-(3), ассоциированных с весовой функцией $\rho(x) \in Q_{1}$, справедлива оценка (4).

2. Пусть $\bar{m}_{0}>0, \bar{m}_{1}>0$ - фиксированные числа. Обозначим через $Q_{1}$ множество всех функций $\rho(x)$, заданных на $[0,1]$ и представимых в виде

$$
\rho(x)=r(x) g(x),
$$

причем предполагается, что функция $r(x)$ является функцией ограниченной вариации и $r(x) \geqslant \bar{m}_{0}$, а функция $g(x)$ - монотонно возрастающая (не в строгом смысле) на [0,1), суммируемая функция, причем $g(x) \geqslant \bar{m}_{1}$ и

$$
\lim _{x \rightarrow 1} g(x)=R \quad(0<R \leqslant \infty) .
$$

Справедлива следующая

Теорема. Пусть $\rho(x) \in Q_{1}$, тогда для собственных функиий $u_{n}(x) \quad(n=1,2, \ldots)$ спектральной задачи (1)-(3) справедлива оченка (4).

3. Доказательство теоремы основывается на леммах 1-4.

Сформулируем эти леммы. 
Лемма 1. Если $\rho(x) \in Q_{1}$, то решение $\varphi(x, \lambda)$ задачи Коши

$$
-\varphi^{\prime \prime}(x, \lambda)=\lambda \rho(x) \varphi(x, \lambda) ; \quad \varphi(0, \lambda)=0, \quad \varphi^{\prime}(0, \lambda)=1,
$$

существует при всех $x \in[0,1]$ и при каждом фиксированном $x \in[0,1]$ является челой функцией параметра $\lambda$.

Фиксируем натуральное число $k$ и собственное число $\lambda_{k}$ спектральной задачи $(1)-(3)$. Собственному числу $\lambda_{k}$ соответствует собственная функция

$$
u_{k}(x)=\left(\int_{0}^{1} \rho(x) \varphi^{2}\left(x, \lambda_{k}\right) d x\right)^{-1 / 2} \varphi\left(x, \lambda_{k}\right) .
$$

Функция $\varphi\left(x, \lambda_{k}\right)$ имеет на $[0,1]$ ровно $k+1$ нулей $z_{j}(j=0,1,2, \ldots, k)$, причем

$$
0=z_{0}<z_{1}<\cdots<z_{k-1}<z_{k}=1 \text {. }
$$

Введем обозначения $(i=1,2, \ldots, k)$

$$
\begin{gathered}
m_{i}=\inf \rho(x), \quad M_{i}=\sup \rho(x), \quad x \in\left[z_{i-1}, z_{i}\right] \\
\delta_{i}=z_{i}-z_{i-1}, \quad h_{i}=\max \left|\varphi\left(x, \lambda_{k}\right)\right|, \quad x \in\left[z_{i-1}, z_{i}\right] ; \\
\alpha_{i}=\left|\varphi^{\prime}\left(z_{i}, \lambda_{k}\right)\right|, \quad \alpha_{0}=1, \quad \tau_{i}=\alpha_{i} / \alpha_{i-1} ; \\
S_{i}=\int_{z_{i-1}}^{z_{i}} \rho(x) \varphi^{2}\left(x, \lambda_{k}\right) d x .
\end{gathered}
$$

Лемма 2. Справедливы неравенства $(i=1,2, \ldots, k)$

Положим для $i=1,2, \ldots, k$

$$
\begin{gathered}
\frac{\alpha_{i-1}}{\sqrt{\lambda_{k} M_{i}}} \leqslant h_{i} \leqslant \frac{\alpha_{i-1}}{\sqrt{\lambda_{k} m_{i}}} ; \quad \frac{\pi}{\sqrt{\lambda_{k} M_{i}}} \leqslant \delta_{i} \leqslant \frac{\pi}{\sqrt{\lambda_{k} m_{i}}} \\
\frac{\pi \alpha_{i-1}^{2}}{2 \sqrt{\lambda_{k}^{3} M_{i}}} \leqslant S_{i} \leqslant \frac{\pi \alpha_{i-1}^{2}}{2 \sqrt{\lambda_{k}^{3} m_{i}}}
\end{gathered}
$$

$$
\begin{gathered}
R_{i}=\sup r(x), \quad r_{i}=\inf r(x), \quad x \in\left[z_{i-1}, z_{i}\right] \\
G_{i} \equiv g\left(z_{i}\right)=\sup g(x), \quad g_{i} \equiv g\left(z_{i-1}\right)=\inf g(x), \quad x \in\left[z_{i-1}, z_{i}\right] .
\end{gathered}
$$

Лемма 3. Справедливы оценки $\left(i=1,2, \ldots, k-1 ; C_{0}>0\right)$ :

$$
\sqrt{\frac{r_{i}}{R_{i}}} \leqslant \tau_{i} \leqslant \sqrt{\frac{R_{i} G_{i}}{r_{i} g_{i}}}, \quad \frac{1}{\sqrt{C_{0}}} \leqslant \frac{\alpha_{i}}{\alpha_{0}} \leqslant \sqrt{C_{0}} \sqrt{\frac{g\left(z_{i}\right)}{g\left(z_{0}\right)}} .
$$

Лемма 4. Справедливо неравенство $(i=1,2, \ldots, k-1)$

$$
\frac{h_{i}}{\left(\int_{0}^{1} \rho(x) \varphi^{2}\left(x, \lambda_{k}\right) d x\right)^{1 / 2}} \leqslant \sqrt{2} \lambda_{k}^{1 / 4}\left(\frac{m_{i}}{\alpha_{i}^{2}} \sum_{j=0}^{k-1} \frac{\pi \alpha_{j}^{2}}{\sqrt{M_{j}}}\right)^{-1 / 2} .
$$

4. Рассмотрим некоторые частные случаи теоремы.

Пусть $g(x) \equiv 1$, тогда $\rho(x) \in Q$, следовательно, доказанная теорема обобщает результат работы [2].

Если же $r(x) \equiv 1$, а $R=\infty$ (число $R$ определено формулой (6)), то мы получим класс $Q_{2}$ весовых функций $\rho(x)$ неограниченной вариации, обладающих тем свойством, что собственные функции спектральной задачи (1)-(3), ассоциированные с весовой функцией $\rho(x) \in Q_{2}$, ограничены в совокупности.

\section{СПИСОК ЛИТЕРАТУРЫ}

[1] Гехтман М. М., Загиров Ю. М., Якубов В.Я. // Функц. анализ и его прилож. 1983. Т. 17. №3. С. 71-72. [2] Гехтман М.М., Айгунов Г.А. // УМН. 1995. Т. 50. № 4 . С. 157-158. [3] Айгунов Г. А. // Известия высших учебных заведений. Северо-Кавказский регион. Естественные науки. 1996. № 1 (93). С. 15-17. 